\section{Clinical Trials in Rehabilita- tion: The Dilemmas and Possible Solutions}

Sir,

Rehabilitation research has gained marked attention in the last two decades. Like other disciplines, rehabilitation practitioners are also required to stay abreast with the latestscientific trends in rehabilitation to make evidence-based decisions. Rehabilitation is a complex health intervention, which has significant implications for the conduct of clinical trials in rehabilitation. ${ }^{1}$

In 2019, the Methods Committee of Cochrane Rehabilitation, in collaboration with the European Journal of Physical and Rehabilitation Medicine, published a special issue on 'methodological problems in rehabilitation research; involving rehabilitation researchers from nine different countries. The special issue highlighted major challenges in the design and conduct of rehabilitation research, using existing models of clinical research and the potential solutions. $^{2}$

The authors would like to emphasise key issues related to clinical trials pertinent to both researchers and clinicians in the field of rehabilitation.

Firstly, in rehabilitation, a typical patient presents with co- or even multimorbidity; and likewise, the treatment is also problem-centred rather than disease-centred. Therefore, it becomes challenging for the clinicians to apply the results of original clinical studies to patients with specific co- or multimorbidity. Meyer and Wulff concluded that majority of the Cochrane reviews on non-specific low back pain (LBP) failed to report on comorbidities or multimorbidity. ${ }^{1}$ Researchers are suggested to consider potential comorbidities during planning, conducting and reporting of clinical trials to reduce bias and increase the generalisability of their findings. ${ }^{1}$

Another key issue is the control group comparisons in rehabilitation trials; the equal provision of factors in complex rehabilitation interventions like communication, clinical competence, and environmental homogeneity across groups is difficult to attain and leads to potential bias. ${ }^{2} \mathrm{~A}$ consensus is required to develop new methods to standardise control conditions in rehabilitation. ${ }^{3}$

Finally, the description of intervention is also vital for clinical applicability, which becomes confusing with the lack of unified terminologies. It is, therefore, recommended to apply the International Classification of Functioning, Disability, and Health (ICF) model to categorise impairments ${ }^{4}$ and the Consensus on Exercise Reporting Template (CERT) to describe interventions ${ }^{5}$. This will allow practitioners to adopt uniform terminologies and standards. ${ }^{4,5}$ The authors believe that in order to improve the generalisability of the clinical trials in rehabilitation, the researchers need to recognise major methodological loopholes before designing a clinical trial; whereas, clinicians should be able to critically evaluate the evidence for validity before making a clinical decision.

\section{CONFLICT OF INTEREST:}

The authors declared no conflict of interest.

\section{AUTHORS' CONTRIBUTION:}

ANM, HT: Substantial contributions to design and concept, literature search, drafting and critical revision; final approval of the version to be published; agreed to be accountable for all aspects of the work in ensuring that questions related to the accuracy and integrity of any part of the work are appropriately investigated and resolved.

\section{REFERENCES}

1. Levack WM, Malmivaara A, Meyer T, Negrini S. Methodological problems in rehabilitation research. Report from a cochrane rehabilitation methodology meeting. Eur J Phys Rehabil Med 2019; 55(3):319-21. doi: 10.23736/ S1973-9087.19.05811-8.

2. Meyer T, Wulff $\mathrm{K}$. Issues of comorbidity in clinical guidelines and systematic reviews from a rehabilitation perspective. EurJ Phys Rehabil Med 2019; 55(3):364-71. doi: 10.23736/ S1973-9087.19.05786-1.

3. Hart T, Bagiella E. Design and implementation of clinical trials in rehabilitation research. Arch Phys Med Rehabil 2012; 93(8):S117-26. doi: 10.1016/j.apmr.2011.11.039.

4. Stucki G, Pollock A, Engkasan JP, Selb M. How to use the International Classification of Functioning, Disability and Health as a reference system for comparative evaluation and standardized reporting of rehabilitation interventions. Eur J Phys Rehabil Med 2019; 55(3):384-94. doi: 10.23736/ S1973-9087.19.05808-8.

5. Hay-Smith EJ, Englas K, Dumoulin C, Ferreira $\mathrm{CH}$, Frawley $\mathrm{H}$, Weatherall M. The Consensus on Exercise Reporting Template (CERT) in a systematic review of exercise-based rehabilitation effectiveness: completeness of reporting, rater agreement, and utility. Eur J Phys Rehabil Med 2019; 55(3):342-52. doi: 10.23736/S1973-9087.19.05791-5.

Arshad Nawaz Malik ${ }^{1}$ and Hina Tariq ${ }^{2}$

${ }^{1}$ Riphah College of Rehabilitation and Allied Health Sciences, Riphah International University, Islamabad, Pakistan

${ }^{2}$ Faculty of Health and Social Sciences, Bournemouth University, UK

Correspondence to: Dr. Hina Tariq, Faculty of Health and Social Sciences, Bournemouth University, UK

E-mail: htariq@bournemouth.ac.uk

Received: March 31, 2020; Revised: April 18, 2020;

Accepted: May 04, 2020

DOI: https://doi.org/10.29271/jcpsp.2021.04.491 